serum HCV VL $>5 \log _{10} \mathrm{IU} / \mathrm{mL}(\mathrm{p}=0.011)$. The magnitude of paired rectal and serum HCV VLs were strongly correlated (correlation coefficient $0.688, \mathrm{p}<0.001$ ). Based on the median quantifiable rectal VL in the absence of visible blood, the surface of an average human penis would be exposed to 2,496 IU of HCV for the duration of anal intercourse.

Conclusion This study provides the first documentation of the presence of $\mathrm{HCV}$ in non-blood rectal fluid. It is plausible that the combination of bathing of an inserted penis in rectal HCV, with the attendant friction of anal intercourse, could result in penetration of HCV into the inserted penis. The protection to the penis afforded by a condom would logically mitigate the risk of transmission by this mechanism. This study should inform public health policy concerning the primary prevention of sexually transmitted HCV.

Disclosure of interest statement There are no competing or financial interests to disclose.

\section{P11.24 LANDSCAPE OF HSV2 AND HIV INFECTIONS AMONG MSMS IN MOROCCO: RESULTS FROM A RESPONDENT DRIVEN SAMPLING SURVEY}

${ }^{1} \mathrm{H}$ Oumzil ${ }^{*},{ }^{1} Y$ Ikken, ${ }^{1} \mid$ Belbacha, ${ }^{1} \mathrm{H}$ Eloudyi, 'S Lemrabet, ${ }^{1} \mathrm{O}$ Bennani, ${ }^{1} \mathrm{R}$ Mengad, ${ }^{2} \mathrm{~A}$ Latifi, ${ }^{3} \mathrm{H}$ Rhilani, ${ }^{3} \mathrm{~K}$ Alami, 'E Elharti. ${ }^{1}$ National Referral Laboratory for HIV, National Institute of Hygiene, Rabat. Morocco; ${ }^{2}$ National Aids Program, Ministry of Health. Morocco; ${ }^{3}$ UNAIDS, Rabat, Morocco

\subsection{6/sextrans-2015-052270.472}

Introduction Over the past two decades, Herpes simplex virus type 2 (HSV2) became the most common cause of genital ulcer in the developed and the developing countries. It represents a risk factor for the acquisition and transmission of other sexually transmitted diseases such as HIV.

With regard to the lack of reports about HSV2 prevalence among Men having Sex with Men (MSMs) in Morocco, this study was conducted to determine the prevalence of the virus among MSMs associated to the risk behaviour.

Methods MSMs from two regions (Marrakech and Agadir) were recruited using Respondent-driven sampling, a chain referral sampling approach. Blood samples collected from 669 recruits were assessed for IgG antibody of HSV2 using ELISA method. Positive samples were tested for IgM to assess recent infection. HIV diagnosis was performed using ELISA test. Reactive samples were confirmed using western bot. Discordant tests were confirmed using PCR. Socio-demographic informations and behavioural data were collected to assess the risk factors associated with infections.

Results The results showed an overall HSV2 sero prevalence of 23\%, and $4.2 \%$ for HIV1 infection. Co-infection with HSV2 among HIV positive recruits was estimated to 50\%. Among HSV 2 reactive recruits, 7\% were positive for HSV 2 IgM, which testifies a recent infection.

Only 22.4\% of MSMs consistently used condoms with male partners and 35\% had never been tested for HIV.

A Risky sexual behaviour and a lack of knowledge about STIs and HIV were the main driver of this situation.

Conclusion Findings suggest continuing STIs risk among MSM in Morocco and a need to strengthen prevention and testing.

\section{P11.25 TIMOR-LESTE NATIONAL SIZE ESTIMATES AND BEHAVIOURAL DATA FOR KEY POPULATIONS AT RISK OF HIV AND OTHER STIS}

${ }^{1} \mathrm{H}_{\text {Jose }}{ }^{*},{ }^{2} \mathrm{P}$ Rawstorne, ${ }^{2} \mathrm{~B}$ Rahman, ${ }^{3} \mathrm{~K}$ Dolan. ${ }^{1}$ Australasian Society for HIV Medicine; ${ }^{2}$ School of Public Health and Community Medicine, UNSW; ${ }^{3}$ National Centre for Drug and Alcohol Research

10.1136/sextrans-2015-052270.473

Introduction The Timor-Leste HIV/STI response is largely focused on the key populations of sex workers, men who have sex with men and transgender (MSM\&TG) and people who use and/or inject drugs (PWUD/PWID). With no prior national size estimation (NSE), a NSE was undertaken to inform HIV/STI programming for these population groups. Behavioural surveying of FSW and MSM\&TG in Dili was also completed.

Methods To improve reliability, several size estimation methods were used: Network Scale-Up; Service Multiplier; Successive Sampling - Size; and 'Wisdom of the Crowd'. Estimates were cross-referenced with service records, estimates from comparable settings and key informant estimates. Plausible estimates were combined and extrapolated to non-assessed districts. A consensus process with key stakeholders was undertaken for validation. For behavioural data, a respondent-driven sampling (RDS)-based survey of FSW and MSM\&TG was conducted in the capital of Dili. Results

1. National size estimates

- FSW: 1688 (95\% CI: 1333, 2044); 0.71\% females aged $15-49$

- MSM\&TG: 8703 (95\% CI: 7821, 9,585); 2.76\% males aged $15+$

- PWUD: 388 (95\% CI: 208, 787); 0.07\% adults aged 15-64

- PWID: 53 (95\% CI: 10, 127); <0.01\% adults aged 15-64

\section{Behaviour}

A total of 125 FSW and 229 MSM\&TG participated in the Dili behavioural survey. Service interaction was mixed, with $67.8 \%$ (95\% CI: $55.7 \%, 79.0 \%, \mathrm{n}=91$ ) of FSW and 51.0\% (95\% CI: $40.6 \%, 61.1 \%, \mathrm{n}=124)$ of MSM\&TG reporting ever having had an HIV test. About one-quarter of both FSW (25.7\%, 95\% CI: $16.1 \%, 34.3 \%, \mathrm{n}=39)$ and MSM\&TG $(26.1 \%, 95 \% \mathrm{CI}$ : $18.8 \%, 33.5 \%, \mathrm{n}=51$ ) reported always using condoms with their client/male sexual partner respectively. Reported illicit drug use among both populations was extremely low.

Conclusion Estimates produced were deemed plausible. The comparably low national PWUD/PWID estimates reflected an extremely low current prevalence of illicit drug use in the country. Behavioural data indicated ongoing HIV/STI risk factors for FSW and MSM\&TG.

Disclosure of interest statement This project received funding from the Ministry of Health Timor-Leste under a grant from the Global Fund to fight AIDS, Tuberculosis and Malaria. No pharmaceutical grants were received in the development of this study.

\section{P11.26 THE SOCIAL AND SEXUAL DYNAMIC BETWEEN GAY MEN/TRANSGENDER PEOPLE AND THEIR STRAIGHT- IDENTIFYING MALE PARTNERS IN TIMOR-LESTE}

${ }^{1} \mathrm{H}$ Jose*, ${ }^{2} \mathrm{P}$ Rawstorne, ${ }^{3} \mathrm{P}$ Gonzaga, ${ }^{2} \mathrm{~S}$ Nathan. ${ }^{1}$ Australasian Society for HIV Medicine; ${ }^{2} \mathrm{School}$ of Public Health and Community Medicine, UNSW; ${ }^{3} \mathrm{~N} / \mathrm{a}$

10.1136/sextrans-2015-052270.474 
Introduction Previous studies in Timor-Leste suggested a unique social and sexual dynamic between self-identifying men who have sex with men (MSM) and their straight-identifying male partners. As part of a national size estimation of key populations at risk, this qualitative study interrogated the dimensions of this dynamic to better inform HIV/STI-related services.

Methods Drawing on ethnographic approaches, semi-structured interviews were undertaken using field notes, including recording of verbatim quotes, with 27 self-identifying MSM, transgender people, straight-identifying MSM and relevant secondary informants across Timor-Leste. Interviews covered gender identity, intra-community social interaction and sexual practices. Data were analysed with involvement of author three (a local researcher who is well-connected to the populations) using an inductive thematic analysis approach where common themes and discrepant cases were coded with attention to the individuals' reported experiences and key events.

Results Three identities among MSM/TG were most commonly reported: self-identifying MSM; transgender; and mane forte (lit.: 'strong man'), or straight-identifying MSM. Self-identifying MSM and transgender-identifying people typically engaged only in sexual activity with straight-identifying MSM. Sex was often reported to have a transactional element, most commonly with MSM or transgender-identifying people providing low-value goods or pocket money to either their casual or long-term straight-identifying male partner/s. An imbalanced power dynamic was often reported between the two parties, with straight-identifying partners generally 'calling the shots' in decisions such as condom use. Straight-identifying men were less likely to interact with MSM HIV/STI services, typically tailored for those identifying as MSM or transgender.

Conclusion The degree to which financial/other incentives play a role in MSM/TG sexual practice is greater than previously reported. The reported power of straight-identifying MSM in sexual decision-making has implications for HIV/STI prevention initiatives, particularly given existing MSM services may not adequately serve straight-identifying MSM.

Disclosure of interest statement This project received funding from the Ministry of Health Timor-Leste under a grant from the Global Fund to fight AIDS, Tuberculosis and Malaria. No pharmaceutical grants were received in the development of this study.

\section{P11.27 DOES ACCESSING NON-OCCUPATIONAL POST EXPOSURE PROPHYLAXIS AND THE ASSOCIATED CLINICAL EXPERIENCE IMPACT CONDOM USE AMONG MEN WHO HAVE SEX WITH MEN IN VICTORIA, AUSTRALIA}

\footnotetext{
${ }^{1,2}$ Anna L Wilkinson*, ${ }^{3}$ Anna B Pierce, ${ }^{1}$ Carol El-Hayek, ${ }^{1}$ Damien McCarthy, ${ }^{3}$ Jude Armishaw, ${ }^{3,4}$ Kerrie Watson, ${ }^{3}$ Brian Price, ${ }^{3,4,5}$ Edwina Wright, ${ }^{6}$ Christopher Fairley, ${ }^{7}$ David Leslie, ${ }^{8}$ Norm Roth, ${ }^{9}$ BK Tee, ${ }^{1,2,10}$ Margaret Hellard, ${ }^{3,4}$ Jenny Hoy, ${ }^{1,2}$ Mark Stoové. ${ }^{1}$ Centre for Population Health, Burnet Institute, 85 Commercial Rd, Melbourne VIC 3000, Australia; ${ }^{2}$ School of Public Health and Preventive Medicine, Monash University, Alfred Hospital, Commercial Rd Melbourne VIC 3000, Australia; ${ }^{3}$ Victorian NPEP Service, Department of Infectious Diseases, The Alfred Hospital, Melbourne VIC 3000, Australia; ${ }^{4}$ Department of Infectious Disease, Monash University, The Alfred Hospital, Melbourne VIC 3000, Australia; ${ }^{5}$ Centre for Biomedical Research, Burnet Institute, 85 Commercial Rd, Melbourne VIC 3000, Australia; ${ }^{\circ}$ Alfred Health, Melbourne Sexual Health Centre, 580 Swanston St, Carlton Vic. 3053, Australia; 'Victorian Infectious Disease Reference Laboratory, 792 Elizabeth St Melbourne Vic. 3000, Australia; ${ }^{8}$ Prahran Market Clinic, Pran Central, Mezzanine Level, Cnr Commercial Rd and Chapel St, Prahran, Melbourne VIC 3181, Australia; ${ }^{9}$ The Centre Clinic, 77 Fitzroy St, St Kilda, 3182; ${ }^{10}$ Alfred Health, Department of Infectious Diseases, The Alfred Hospital, Melbourne VIC 3000, Australia
}

Background Condom promotion is a key component of combination HIV prevention globally. There is evidence from clinical settings that intense interventions successfully increase condom use. However it is unknown if routine sexual health clinical encounters, including encounters potentially prompted by concern relating to particularly high risk events, have the same impact on reducing HIV risk behaviours. We compared selfreported condom use among MSM before and after receiving standard care non-post exposure prophylaxis.

Methods Data from MSM accessing the Victorian NPEP Service (VNPEPS) was linked to HIV/STI testing data from Victorian Primary Care Network for sentinel surveillance (VPCNSS) clinics between 2007-2013. Analysis included data from MSM who accessed NPEP and reported condom use at their most recent HIV/STI test prior to NPEP (baseline) and two tests following NPEP (follow-up one and two). Only the first NPEP episode was included. Proportion of MSM reporting inconsistent condom use at baseline (test immediately prior to NPEP) and follow-up one and two were compared using a two sample test of proportions.

Results Among 1199 MSM presenting for NPEP on 2094 occasions, 6329 test and risk behaviour records were obtained from VPCNSS sites pre-and post-NPEP. A total of 303 MSM had data on condom use at baseline and two follow ups. Inconsistent condom use was reported by 146 (48.2\%) of MSM at baseline, 138 $(45.5 \%)$ at follow-up one $(p=0.60)$ and $146(48.2 \%)$ at follow-up two $(\mathrm{p}=1.0)$. Follow-up two occurred a median of 15 months (IQR $=10-23)$ after NPEP presentation.

Conclusion In this study we found no change in condom use following NPEP among MSM with pre- and post-NPEP VPCNSS testing histories. Though generalisability to all MSM is limited, this analysis offers insight into a key risk population and highlights the potential need for tailored strategies to promote primary prevention during risk event-prompted clinical presentations.

Disclosure of interest statement The Victorian Department of Health funds the VNPEPS and ongoing surveillance projects within the Burnet Institute. The authors would like to acknowledge the NHMRC who provide funding to Margaret Hellard as a Senior Research Fellow, Mark Stoové as a Career Development Fellow and Anna Wilkinson as a public health scholarship recipient. Edwina Wright receives funding from a research grant from $\mathrm{NIH}$, research funding from the Victorian Department of Health and unrestricted research funds from Gilead, Abbott, Janssen Cilag, MSD and Boehringer Ingelheim. She has also received funding that has been used for research purposes only from ViiV, Merck, Gilead, and Abbott for consultancy work, payment for lectures from ViiV and payment for developing educational resources for ViiV, MSD and Gilead. The study drug for the VicPrEP study has been donated by Gilead Sciences. The authors gratefully acknowledge the contribution to this work of Victorian Operational Infrastructure Support Program received by the Burnet Institute. 